\title{
Transatlantica
}

Revue d'études américaines. American Studies Journal

$2 \mid 2014$

Aesthetics of Theory in the Modern Era and Beyond / Photographie documentaire

\section{Colloque international « Survivance des icônes, usage et recyclage des figures et emblèmes culturels (XX' ${ }^{\mathrm{e}}-\mathrm{XXI}^{\mathrm{e}}$ siècles) »}

Université Paris-Dauphine et ENS, 4 et 5 avril 2014

Juliette Mélia

\section{OpenEdition}

Journals

Electronic version

URL: https://journals.openedition.org/transatlantica/7066

DOI: 10.4000/transatlantica.7066

ISSN: 1765-2766

\section{Publisher}

Association française d'Etudes Américaines (AFEA)

\section{Electronic reference}

Juliette Mélia, "Colloque international « Survivance des icônes,

usage et recyclage des figures et emblèmes culturels (XXe-XX|e siècles) »", Transatlantica [Online], 2 I

2014, Online since 31 December 2014, connection on 04 February 2023. URL: http://

journals.openedition.org/transatlantica/7066 ; DOI: https://doi.org/10.4000/transatlantica.7066

This text was automatically generated on 4 February 2023.

\section{c)}

Creative Commons - Attribution-NonCommercial-NoDerivatives 4.0 International - CC BY-NC-ND 4.0

https://creativecommons.org/licenses/by-nc-nd/4.0/ 


\section{Colloque international « Survivance des icônes,}

\section{usage et recyclage des figures et emblèmes culturels $\left(\mathrm{XX}^{\mathrm{e}}-\mathrm{XXI}^{\mathrm{e}}\right.$ siècles)»}

Université Paris-Dauphine et ENS, 4 et 5 avril 2014

Juliette Mélia

Organisées par Géraldine Chouard (Professeure de civilisation américaine, Université Paris-Dauphine) et Déborah Lévy-Bertherat (Maître de Conférences en littérature comparée, ENS Ulm), ces deux journées de colloque international s'inscrivent dans le cadre de «Paris Sciences et Lettres» (PSL) et sont une manifestation de l'union entre les deux établissements partenaires. Ce colloque fut celui du mélange des genres, des registres, de la création d'une synergie faite des improbables rencontres de Babar et de Staline, de Mickey et de Mapplethorpe, de La Fille de Dracula et de Peau d'Âne, et bien d'autres... Son intitulé, "Survivance des icônes ", est une référence à l'ouvrage de Georges Didi-Huberman, Survivance des lucioles (2009), qui déplore les effets d'une modernité qui tue les lucioles. Mais la survivance des lucioles, c'est aussi le contrepouvoir, la vitalité intellectuelle qui se manifeste encore plus vivement dans les périodes de malaise, de crise. Les icônes sont-elles des lucioles métaphoriques? Sontelles des références aveuglantes ou des phares culturels qui nous guident dans un domaine inconnu, au risque de nous éblouir par l'effet du lieu commun, l'adhérence obligée, sans recul critique possible, qu'elles impliquent? 


\section{Atelier 1 : « Icônes et arts visuels »}

\section{Richard Phelan (Université Aix-Marseille), "Le temps des icônes : ce que durent les figures et emblèmes culturels »}

Richard Phelan a proposé une introduction au colloque en offrant une méthodologie d'analyse du mot « icône ». L'étymologie du terme est le grec icona, qui veut dire image, mais aussi stéréotype socioculturel. La métaphore se dégage du sens : l'icône est image de quelqu'un ainsi qu'objet de dévotion. Un exemple d'icône est la figure de la piéta, c'est pourquoi la ville de Soweto fut à certaines périodes saturée par cette icône, symbole du pouvoir demandé par les femmes d'Afrique du Sud, subissant les intimidations de leurs maris et du régime. Les critères des icônes sont leur grandeur, leur stabilité. Selon Richard Phelan, une analyse des archives du journal Le Monde peut nous le confirmer. La lecture des articles comportant le mot «icône " montre que le terme n'est pas forcément abusif : "l'icône du rap trash » dit clairement l'étendue de son public, et quiconque n'étant pas versé dans le rap trash ne reconnaîtra pas la valeur d'icône du sujet présenté. Ces analyses d'archives mettent aussi au jour des abus, des surprises. Bob Woodward, par exemple, n'est pas une icône du journalisme, c'est un abus de langage de parler d'icône à son propos. Les domaines d'usage du terme «icône » sont variés : la politique, avec par exemple Nelson Mandela dont la vie et la mort seront des instances iconiques, le cinéma bien sûr, les produits manufacturés, la peinture et la photographie, avec Warhol, la musique, avec David Bowie en icône d'un dandysme sulfureux, le sport, la mode, la télévision, l'architecture, le monde des affaires... On remarque également que les icônes vont souvent par deux ou par trois, car c'est en tant que réseau qu'elles sont le plus visibles: par exemple, Mandela, Martin Luther King et Gandhi sont souvent associés. Richard Phelan conclut en disant qu'il nous reste à nous interroger sur la destination, l'utilité des icônes. Pour qui, pourquoi y a-t-il des icônes?

\section{Juliette Melia (Université Paris 7), « La farce de l'iconographie chrétienne dans les autoportraits de Robert Mapplethorpe »}

Selon l'analyse de Juliette Melia, l'incarnation de Robert Mapplethorpe dans sa photographie peut correspondre à trois mouvements : revalorisation, humour, puis humour noir qui mélangent les iconographies, entre glamour homosexuel et cliché chrétien utilisé avec ironie. Un autoportrait célèbre de 1975 nous renvoie au supplice chrétien par excellence, celui de la crucifixion, dans une iconographie qui relève bien de la farce, du comique, avec le mélange des genres entre sacré et profane. Un autre autoportrait, daté de 1978, mettant en scène un fouet, est très sulfureux. En quoi relève-t-il d'une iconographie chrétienne? Ici Mapplethorpe semble s'être inspiré du cliché du diable, le fouet pouvant être une métaphore visuelle de sa queue fourchue. Les années 80 , avec la montée du conservatisme, le SIDA, font un contexte particulier à la dernière phase des travaux de Mapplethorpe, qui deviennent plus contestataires. Dans un autoportrait ou son visage est surmonté de deux petites cornes (une façon de dire «I'm horny » ?), le regard est plein d'un sentiment de défi, anticipant les analyses de Judith Butler sur la resignification de l'insulte comme acte de liberté par excellence. Ici, Mapplethorpe reprend avec ironie les thèses homophobes de Jesse Helms qui fait de 
l'homosexualité un fléau moral et les transcende en une œuvre d'art. Si l'esthétique catholique transparait dans nombre des œuvres de Robert Mapplethorpe, quand il s'agit des autoportraits, l'utilisation du symbolisme chrétien fait passer la représentation dans un domaine ambigu, entre vérité et mascarade, entre manipulation et révélation de l'identité. Les rôles qu'il joue créent dans l'esprit du spectateur un phénomène de connivence, d'autant plus que les symboles sont pervertis, les icônes sont réinterprétés. Voilà ce qui donne aux autoportraits de Mapplethorpe leur humour inattendu, et par cet humour qui implique forcément le spectateur, la portée politique qui a valu à l'œuvre d'être au centre des Culture Wars après sa mort.

\section{Jean-Paul Gabilliet (Université Bordeaux-Montaigne), « Pastiches et détournements de Mickey Mouse dans les comix de l'âge psychédélique (1966-1976)»}

4 Jean-Paul Gabilliet nous rappelle que Mickey Mouse est l'icône par excellence de la culture américaine, l'emblème d'un système de valeur dont il est devenu synonyme avec sa valeur adjectivale: on l'emploie pour dire "médiocre, de peu de valeur, mesquin... ». Au sein de la culture américaine, il est l'icône d'une certaine culture moyenne qui prône l'euphémisation des problématiques, comme le veut la culture mainstream des années Eisenhower. À l'âge adulte, la génération des enfants qui a grandi en regardant les dessins animés de Mickey participe à la création contreculturelle, bouillonnement créatif sans précédent de contestation et de réécriture des valeurs, de subversion des symboles culturels dont la petite souris fait les frais. Une des publications qui utilise le plus la figure de Mickey est le magazine Mad, qui fait de Mickey un personnage récurrent en tant que symbole d'une idéologie rejetée. La subversion de la figure mousienne peut prendre diverses formes, comme le détournement métonymique, quand un élément reconnaissable est utilisé (les oreilles, le gant...), ou le détournement psychédélique, lorsque le code visuel de Mickey est métamorphosé et qu'il est sexualisé par exemple. Le détournement peut aussi être parodique et la subversion se baser plus sur la narration que sur l'image, ou passer par le personnage parodique de Mickey Rat, générant des procès, le risque de confusion entre les deux Mickeys étant vécu par les ayant-droits comme un crime de lèse-majesté culturel, de même que les tentatives de représentation de la sexualité de Mickey. Si la résilience des icônes était à prouver, on noterait que l'aura de Mickey Mouse n'est pas affectée par les parodies, au contraire. La puissance de son image fait d'elle de nos jours une métonymie de l'ensemble du système Disney, et on trouve des références à l'emprise culturelle du Disney Mickey Mouse Club jusque dans des films de guerre comme Full Metal Jacket, de Stanley Kubrick en 1986, où des soldats partent au combat en chantant le générique de l'émission télévisée. 


\section{Atelier 2 : Icônes et politique}

\section{Daria Sinichkina (ENS-Université Paris 4), « D'ennemis du peuple à martyrs du stalinisme : la réception des écrivains victimes de la terreur dans les années 1980 et 1990 »}

5 Les poètes peuvent-ils devenir des icônes, s'est interrogé Daria Sinichkina, et si oui, comment et pourquoi ? Lors de la pérestroïka, on assiste à un boom éditorial, qui soulève la question de comment s'est opéré le retour des écrivains sur la scène publique après le communisme. Dans les années 1987-89, la mémoire du stalinisme est encore vive, et la population est dans une fièvre de lecture car la censure prend fin à l'époque de la glasnost, et les lecteurs fascinés par l'historique de la période précédente peuvent accéder aux œuvres précédemment interdites, comme celles des poètes du début du siècle, appelé "âge d'argent ", certains de ces poètes ayant connu un destin tragique. On assiste ensuite à une période de zsezda, c'est-à-dire de critique de l'âge d'argent. Les années 1989-91 voient une deuxième vague de réhabilitation éditoriale. L'intérêt pour le stalinisme décroit, au profit d'une crise d'identité de la population russe, qui idéalise la période prérévolutionnaire. C'est une des différences entre la culture russe officielle et la culture russe véritable. Ce sont les magazines littéraires qui ont une circulation extraordinaire à cette période, qui montrent les poètes comme des icônes dans des portraits photographiques de ces derniers aux visages émaciés, aux souffrances passées lisibles dans leurs yeux hagards au sortir de la zsezda. La torture est manifeste dans les images, les hommes de lettres sont bien érigés en martyrs par la culture russe populaire. Comment réagira l'appareil d'état, le KGB ? À la fin de la pérestroïka, on assiste à une nouvelle période d'anarchisme éditorial, et ce sont les statues dressées à cette époque pour les poètes qui prennent une nouvelle importance dans l'établissement de leur iconicité.

\section{Martine Piquet (Université Paris-Dauphine), « L'icône du digger [poilu] dans la culture australienne »}

6 Martine Piquet s'est interrogée sur la manière dont le digger, soldat australien de la Première Guerre Mondiale, a accédé au statut d'icône célébré par de marquantes commémorations dans la société australienne. Le poème de Laurence Binyon, «For the Fallen » (poème écrit en 1914), peut être un facteur déclencheur du phénomène. Il ne remémore pas la victoire, mais la bataille de Gallipoli, un des événements les plus calamiteux de la Première Guerre Mondiale. La bataille de Gallipoli est importante car c'est elle qui cristallise un sentiment national, comme en témoigne le texte lyrique d'Ellis Ashmead, qui fait de la guerre un rite de passage non seulement pour l'homme australien, mais aussi pour la nation australienne. En 1915, le poème de Banjo Paterson, "We're All Australian Now ", témoigne d'une opinion publique enthousiaste en faveur de la guerre, avec beaucoup d'enrôlements volontaires, parfois illégaux. Quelles sont les motivations de ces jeunes hommes qui s'engagent? L'Australie est toujours une colonie, fédérée en 1901, ce qui lui donne une identité duelle. Le magazine The Bulletin met en scène des héros de guerre selon une typologie assez stable, des identités claires et rassurantes. Il y a entres autres le swagman, l'homme au balluchon, le digger, l'orpailleur, puis par extension le poilu, le dinkum Aussie, le dur à cuire de l'outback. Un 
autre facteur de motivation pour les jeunes qui s'enrôlent est l'iconisation du Private Simpson, un jeune brancardier mort au front et qui devient une légende commémorée par force statues, timbres, médailles, figurines pour enfant... L'image du Private Simpson sublime les qualités nationales australiennes. La figure du digger a-t-elle un rapport de cause et/ou de conséquence dans la question de sentiment national ? Le digger n'est-il qu'un héro folklorique ? Quel rôle ont les soldats dans l'invention de ce personnage par rapport au rôle de l'historien officiel ? L'Australien aime à se décrire comme le summum de la masculinité, est-ce lié à la vantardise de ces soldats ? Walzing Matilda et d'autres chansons populaires ont-elles contribué à l'iconisation du soldat? Les femmes sont-elles concernées par cette icône qui semble les exclure ? Pour résumer l'iconicité du digger, ce sont des hommes qui préfèrent mourir que se rendre, qui glorifient leur esprit de rébellion, valeurs qui se retrouvent dans le sentiment national australien.

\section{Zoé Carle (Université Paris 3), « La martyrologie de la révolution égyptienne : des images aux icônes »}

7 Zoé Carle a basé sa conférence sur des événements très concrets de l'histoire égyptienne récente : le 6 juin 2010, dans la ville d'Alexandrie, en Égypte, Khaled Said meurt sous les coups de policiers. Son visage tuméfié devient l'emblème de la brutalité policière, grâce à sa diffusion virale sur les réseaux sociaux. « Nous somme tous Khaled Said » devient un leitmotiv de la jeunesse égyptienne, le slogan des manifestations au Caire et à Alexandrie avant janvier 2011, renforçant encore l'énorme impact de cette photo. Le gouvernement égyptien consent à faire juger les deux policiers responsables, mais pas avant que ne se soit créé un réseau de symboles, par la démultiplication des images, qui font de Said un martyr, hybride de saint et de héros national. En effet, sa photo se multiplie à toute vitesse dans l'espace public, sous forme de graffitis (qui peuvent devenir des sanctuaires éphémères), masques pour manifestants, et il devient une figure de rassemblement pour un peuple égyptien en mal de figure charismatique. À partir de juin 2012, Khaled Said n'est plus le seul visage de la révolution égyptienne, d'autres martyrs apparaissent alors que se succèdent les affrontements, comme Gika, premier martyr de l'ère Morsi (on entend parfois l'expression « Tu es un vrai Gika »). La révolution est une sorte de nouveau patrimoine national, l'enjeu est fort, il s'agit de revendiquer pour son camp la bonne version de la révolution du 25 janvier. À ce titre, les captations symboliques des martyrs sont un enjeu important, sur le plan symbolique, économique, juridique. Il s'agit de convaincre que le martyr est bien mort pour les bonnes idées. De plus, il existe un statut de «martyr de la révolution » qui garantit à la famille une compensation financière et une aide juridique, et permet l'ouverture d'une enquête. Une question se pose, celle des conséquences de la rapidité de circulation des icônes via les nouveaux espaces numériques investis par la jeune génération. On assiste à une accélération de la temporalité : chaque nouvelle image chasse la précédente. Une forme de myopie historique se crée donc, qui occulte les événements précédents. Les visages de Said et Gika sont représentés de manières très différentes : visage du supplicié d'un côté, visage en gloire, en majesté de l'autre. Est-ce incompatible avec une culture musulmane qui interdit la représentation de la figure humaine? La transgression de cet interdit montre un autre aspect de l'importance de l'icône : celle-ci peut aussi être laïque, l'imagerie religieuse étant peu compatible avec les revendications plutôt libérales des manifestants. 


\section{Atelier 3 : « Icônes et culture populaire »}

\section{Claude Chastagner (Université Montpellier 3), « Iggy Pop, le marketing de la rébellion/transgression »}

Claude Chastagner aborde Iggy Pop non pas au prisme de sa carrière musicale, mais bien par le biais de sa persona, sa valeur d'icône en évolution. En effet, Iggy Pop est surtout connu pour ses excès divers, il est un signe, il signifie le rock en adoptant des comportements extrêmes, refusant le contrôle que les autorités imposent à l'individu pour le transformer en citoyen obéissant. Son corps est en jeu, ses excès impliquent le corps, et sont le signe d'une totale liberté, indépendance et émancipation par rapport aux classes dominantes, même si c'est un rôle et pas une façon de vivre au quotidien, comme en témoignent de nombreuses publicités où figure Iggy Pop, dans lesquelles il joue avec les idées de défamiliarisation, de transgressions enchâssées dans une logique commerciale. On peut se demander si cela régénère ou affaiblit la transgression. D'une part, la récupération par le marché effectue une dilution, un affadissement de la valeur rebelle d'Iggy Pop, générant un regard méprisant sur l'icône rebelle ayant vendu son corps au marché. D'autre part, Iggy Pop se sert d'une forme ambigüe de l'économie de marché pour pouvoir utiliser ses propres valeurs transgressives et provocatrices, qui sont les mêmes valeurs que le capitalisme contemporain, qui n'est plus un ordre rigide et patriarcal, mais prône de nouvelles valeurs et stratégies, comme l'hédonisme et la satisfaction immédiate des désirs. Le recyclage de la figure du rockeur âgé dans le marketing est une forme encore plus radicale de transgression. On peut s'attarder sur le ridicule de ces stars vieillies et mercenaires, faisant l'exhibition vénale de leur corps. Mais cela peut aussi être interprété comme la dernière des insolences, un doigt d'honneur fait aux conventions, la transgression obligée par les lois du rock, non plus soumission mais rébellion. Quant au fait d'afficher ses stigmates, cela peut être lu comme une déchéance pitoyable, mais aussi comme un refus audacieux, et typique de l'esprit rock, des impératifs esthétiques de notre époque et son lot de chirurgie esthétique et de retouche photographique. Alors que les années 60 fétichisaient la jeunesse, notre époque refuse le diktat de l'âge. Une autre caractéristique de notre époque est le refus de la séparation public/intime, qui modifie les codes de représentation. Peut-être est-ce là que repose le réel iconoclasme ? En tout cas, cette invasion de l'intime dans la sphère publique influence notre perception de l'âge et du vieillissement. Dans ce domaine, Iggy Pop joue avec le feu, toujours à la limite du refus et de l'acceptation.

\section{Émile Bertherat (Université Paris 1), « Les icônes dans le cinéma de Jacques Demy »}

9 Émile Bertherat s'est attaché au cinéaste Jacques Demy car il est l'un des rares cinéastes français ayant généré des icônes, en particulier pour ses films des années 60 (Lola, Model Shop, Peau d'Âne etc.) comportant beaucoup de situations et personnages si récurrents qu'ils en deviennent iconiques. À l'origine, les films de Jacques Demy sont influencés par la peinture et les icônes culturelles. Par exemple, Les Parapluies de Cherbourg ont été comparés à " un Matisse qui chante », et Peau d’Âne se base sur des gravures de Gustave 
Doré, pour la mise en scène figée des personnages, leur composition picturale, leurs plans fixes. Jacques Demy utilise également des univers colorés homogènes pour créer une iconicité, comme les royaumes rouges et bleus dans Peau d'Âne, ou la figure du marin (qui est une autre icône récurrente, apparaissant dans Lola, Les Demoiselles de Rochefort). Les influences romanesques, cinématiques, picturales du cinéaste passent toutes dans son prisme, même son rapport intime à la ville de Nantes, dont il filme le désormais célèbre Passage Pommerel dans Lola et Les Parapluies de Cherbourg, dans un intéressant cas de ville-icône. À Nantes, Demy est une icône, il existe un "tour Demy " pour visiter les lieux les plus cinématographiques de la ville. Mais le personnage phare de Demy est bien sûr Catherine Deneuve, transformée en icône par sa caméra. Demy lui donne son premier rôle important dans Les Parapluies, un rôle où elle est très valorisée, l'objet de tableaux à l'intérieur des films. Pourtant, ses personnages sont toujours désenchantés, mélancoliques comme dans Les Parapluies ou en fuite comme dans Peau d'Âne. À leur tour, les icônes de Jacques Demy sont ensuite recyclées par sa femme et son fils, Agnès Varda et Matthieu Demy, qui mettent en scène des photos reprenant les situations du film, et qui sont peut-être les auteurs de la recette du « cake d'amour » de Peau d'Âne postée sur le site de recettes marmiton.org. Tout le monde reconnaît certaines images des films de Jacques Demy, c'est en cela qu'elles sont iconiques.

\section{Fabrice Lyczba (Université Paris-Dauphine), « 'When Icons Walked the Earth' : un Tarzan dans la ville, 1920-1921»}

10 La question originale posée par Fabrice Lyczba est celle du son : un son peut-il aussi être iconique? Le cas de figure retenu sera celui du cri de Tarzan, ou plutôt du yodel de Johnny Weissmuller, utilisé par la suite dans toutes les adaptations de Tarzan. Le cri et le langage de Tarzan sont sujets à controverse. En effet, les romans originaux ne disent pas clairement quel est son langage. Fabrice Lyczba est revenu sur le mythe de Tarzan : ce dernier est un moyen de se réapproprier une vraie masculinité. Si les déguisements de Tarzan sont puérils, de simples objets marketing pour les enfants, ils font néanmoins partie d'un ensemble cohérent : le désir de prolonger la fiction au-delà de l'écran de cinéma. Par exemple, les halls de cinémas étaient ornés de vrais palmiers qui empêchaient presque l'accès aux salles, puis dans ces dernières, avant le début du film, d'autres mises en scènes compliquées telles que des ballets. Enfin, un canular fut publié dans un journal, selon lequel Tarzan vivait bel et bien dans une chambre d'hôtel de New York avec un lion vivant. Tous ces éléments finirent par créer un regard particulier chez les spectateurs de Tarzan, qui voyaient dans ce personnage plus qu'un personnage ordinaire. De nos jours, Tarzan est une icône culturelle, qui représente le passage du monde sauvage au monde civilisé, une façon de démontrer l'emprise des médias face à ce personnage à l'intéressant statut, entre mythe et icône.

\section{Victoria Amador (American University of Sharjah, Arab Emirates), "The Vampire as a Global Icon"}

La conférence de Victoria Amador prend sa source dans la récente reconnaissance par l'université de l'engouement pour l'étude du gothique. En effet, il apparaît depuis peu que le vampire est présent dans toutes les cultures - ce serait l'une des croyances les plus anciennes. De plus, les vampires sont décrits comme une invasion : ils symbolisent 
la peur de l'immigration, expriment une vision xénophobe que l'Occident aurait de l'Orient. La littérature britannique du XIXe siècle comportait initialement de nombreux vampires féminins où la femme est dans un rôle dominant. La femme-victime est apparue dans les romans gothiques postérieurs. Le vampire est également devenu de plus en plus sexualisé, une façon de faire ressentir aux jeunes lectrices des émotions défendues. Carmilla, de Sheridan Le Fanu (1872), mettait par exemple en scène une vampire lesbienne qui était un personnage attachant, complexe, aussi bien victime que bourreau. Le vampire donnait une nouvelle image de la femme - dangereuse, prédatrice, indifférente - et représentait la peur de la sexualité et du pouvoir féminins. Les vampires sont friands d'action et de jeunesse, ce qui pourrait expliquer leur migration vers les États-Unis. En effet, les personnages féminins d'Edgar Allan Poe ont souvent des caractéristiques vampiriques, et $\mathrm{T}$. $\mathrm{S}$. Eliot aborde le mythe de la femme vampire dans «The Waste Land» («La Terre Vaine »). Le XX ${ }^{\mathrm{e}}$ siècle fut le siècle du triomphe de la femme et de l'Amérique, et les femmes deviennent capables de s'approprier le mythe du vampire, mais aussi du monstre et d'autres personnages dangereux, comme le fait l'actrice Theda Bara ou la flapper, jeune femme moderne aux cheveux courts et aux mœurs libres, icône des années 20, femme affirmée, dangereuse et irrésistible. Un exemple cinématographique pourrait être La Fille de Dracula, de Lambert Hillyer (1936), dans lequel l'actrice principale, Gloria Holden, séduit aussi bien les hommes que les femmes. En tant qu'icône mort-vivant, le vampire peut faire l'objet d'une redécouverte perpétuelle, et son iconicité peut être éternelle. Les vampires nous fascinent car ils nous ressemblent, en plus beaux, plus désinhibés, dotés de la jeunesse éternelle à laquelle la société nous fait aspirer. Selon Victoria Amador, les vampires sont nous-mêmes.

\section{Table Ronde}

\section{Anne Crémieux (Université Paris-Ouest) et Georges-Claude Guilbert (Université de Tours), «Iconicité LGBTQI : pistes épistémologiques, dimensions esthétiques et aspects politiques »}

Pour Anne Crémieux et Georges-Claude Guilbert, plusieurs questions se posent à propos d'iconicité LGBTQI. Que veut dire le «T»? On peut le remplacer par "Trans'", mais est-ce synonyme de transgenre, transsexuel ou une autre réalité ? Pourquoi y-a-t'il beaucoup d'icônes pour les hommes homosexuels? Pourquoi est-il difficile de nommer une icône intersexe ? On assiste souvent à un phénomène d'iconisation des icônes gays par certains photographes ou cinéastes. Les thématiques sont parfois religieuses, comme chez David Lachapelle ou Robert Mapplethorpe, ou camp. Les documents qui décortiquent le phénomène sont le film The Celluloid Closet (1995), ou l'ouvrage Making Things Perfectly Queer (1993).

Puis, Anne Crémieux et Georges-Claude Guilbert ont passé en revue un grand nombre d'exemples : Mae West fait preuve d'un humour masculin, elle écrit des pièces jouées par des hommes travestis; Agnes Moorehead a des comportements et des tenues de drag queen; Marlene Dietrich se caractérise par son hyperféminité, on la décrit parfois comme " male and female drag "; Tallulah Bankhead est l'incarnation du camp; Frida Kahlo est une icône bisexuelle récupérée par les lesbiennes, bien que tardivement, pour sa personnalité, son physique, et sa peinture ; Bette Davis ; Carmen Miranda, dont la 
personnalité comporte une grande composante de travestissement ; Judy Garland, qui a donné lieu à l'expression "a friend of Dorothy's » désignant un homosexuel (Garland, quant à elle, est l'archétype de la "fag hag ", la femme hétérosexuelle fascinée par les homosexuels, ce qui ressort de plusieurs de ses mariages à des homosexuels); Marilyn Monroe, en particulier pour son rôle dans Some Like it Hot avec Jack Lemon et Tony Curtis travestis; Sara Montiel, une icône gay espagnole; Sheila, l'icône gay des pauvres; Lady Gaga, qui chante Born This Way, offrant une lecture essentialiste de l'homosexualité ; Jacky Kennedy ; Liz Taylor ; Dalida ; Joan Rivers ; Joan Collins ; Jacky Collins ; Cher ; Annie Lennox. Quant aux icône gays masculines, on peut citer les superhéros dans leur ensemble, Oscar Wilde, Marlon Brando, Truman Capote, Andy Warhol, Bambi, qui a des caractéristiques transgenre, Tim Curry, Marie France, une célèbre transsexuelle française. À propos des icônes lesbiennes, Anne Crémieux et GeorgesClaude Guilbert remarquent qu'il y a peu d'identification à des personnages non lesbiens. Les exemples cités furent Chevalier d'Eon, Wonder Woman, élevée par des Amazones, Gertrude Stein, Natalie Barney, Agnes Morrehead, Greta Garbo, surtout dans son rôle de Reine Christine, Katherine Hepburn, Barbra Streisand, Muriel Robin, Jamie Lee Curtis (la rumeur la veut intersexuée, du fait de sa grande taille, sa minceur, sans oublier ses personnages qui se trouvent dans ce qu'on appelle "the glass closet", quand l'homosexualité est connue de tous mais que le coming out n'est pas officiellement fait).

\section{Samedi 5 avril 2014}

\section{Conférence Plénière}

\section{Jean Kempf (Université Lyon 2), « Icônes américaines : entre anonymat et reconnaissance/une histoire personnelle »}

14 Le postulat de base que nous a présenté Jean Kempf en conférence plénière est volontairement paradoxal : il ne peut pas y avoir d'icône purement personnelle, l'icône est une société qui vous traverse. Jean Kempf s'appuie sur une série de photos qu'il appelle «images sans qualités", par analogie avec L'Homme sans qualités de Robert Musil, images dont la valeur n'est pas intrinsèque mais vient du fait qu'elles sont traversées par la culture américaine, qu'elles sont l'expression d'un contact des cultures entre un américaniste et l'Amérique elle-même. La mise en abîme, l'image dans l'image, un classique du genre photographique, est un trope important de la série, et se sert de l'image insérée pour créer l'iconicité. Celle-ci naît aussi du fait de photographier des images iconiques de la culture américaine, comme le Plymouth Rock, le galet qui est censé être la roche même sur laquelle les Pères Pèlerins ont posé le pied. Une image d'un armurier est une référence à Berenice Abbott, celle du célèbre bâtiment surnommé Flat Iron est une reprise de Stieglitz et de Steichen. Jean Kempf voit les États-Unis à travers leurs photographes, la médiatisation des États-Unis étant telle qu'on arrive, dès qu'on parcourt le territoire américain, forcément dans des lieux qui rappellent une série, un film, des photographies. Surmédiatisé, le paysage américain lui-même est devenu iconique, comme si les lieux étaient devenus des lieux communs. 


\section{Atelier 4 : « Grands témoins du siècle »}

\section{Guido Furci (Université Paris 3), «Primo Levi, l'homme et l'icône »}

15 Guido Furci a analysé la place des icônes au sein d'une société capitaliste avancée, à travers le film d'animation Le Congrès d'Ari Folman (2013), qui dépeint un monde d'hallucination permanente, et plus particulièrement l'utilisation iconique du personnage de Primo Levi, dont il est fait une caricature qui s'inspire d'une représentation codifiée de l'auteur (tenue de travail de chimiste, lunettes...), ce qui soulève la question suivante : pourquoi rendre ce personnage si complexe par la bidimensionnalité du dessin? Un certain nombre de clichés sont souvent utilisés pour représenter Levi : les lunettes, la cigarette « existentialiste ». Les lunettes de Levi sont comme une métonymie du personnage. L'exemple de Guido Furci montre comment l'iconicité d'un acteur de l'histoire peut le transformer en image, en objet de consommation visuelle.

\section{Atelier 5 : « Icônes de la culture de jeunesse »}

\section{Isabelle Nières-Chevrel (Université Rennes 2), « Et voilà pourquoi Babar porte (toujours) un costume de couleur verte »}

Isabelle Nières-Chevrel s'est interrogée sur le mode d'accès au statut d'icône pour un personnage de livre pour enfants, au travers de la création de Jean de Brunhof, le héro hautement positif de Babar. Le vert vif du costume n'a-t-il été choisi que pour des raisons d'harmonie chromatique, pour complémenter le gris de la peau? Ou parce que cette couleur est inédite pour un costume et lui donne une individualité ? Laurent de Brunhof reprend le personnage créé par son père à la mort de ce dernier, ce qui peut expliquer le destin dégradé du personnage de Babar, qui devient une marque. Pourtant, malgré cette déchéance mercantile, le costume vert demeure car il permet à l'enfant de reconnaître immédiatement le personnage. Mais plus que ça, l'habit est un enjeu: Babar, l'éléphant anthropomorphe, relève-t-il de l'animalité ou de l'humanité ? Le costume, symbole de civilisation, le place d'emblée dans le monde des hommes, sans sacrifier à la nature, à une certain petit côté loufoque grâce à la couleur verte. Costume vert, couronne simplifiée jaune, peau grise : par son minimalisme iconique, Babar est l'incarnation de ce que la France peut produire de rassurant.

\section{Mathilde Lévêque (Université Paris 13), « Des icônes tirées par les cheveux : figures iconiques de l'enfance aux $\mathrm{XX}^{\mathrm{e}}$ et $\mathrm{XXI}^{\mathrm{e}}$ siècles »}

Mathilde Lévêque a choisi dans sa communication de passer par le prisme de la chevelure pour analyser l'icône culturelle de l'enfance. Shirley Temple, par exemple, est l'enfant-icône de l'Amérique des années 30, comme en témoignent ses produits dérivés innombrables. Dans le contexte de crise et de grande dépression des années 30, elle est un symbole d'optimisme et de courage, avec l'effet iconique de ses boucles en forme de ressorts, métaphores d'un dynamisme américain retrouvé/retrouvable. Elle joue d'ailleurs beaucoup de rôles de petites filles pauvres récompensées à la fin du film. On peut opposer Temple à Jackie Coogan, l'enfant qui a joué dans Le Kid avec Charlie 
Chaplin, et établir une dichotomie genrée, la petite fille bouclée sage d'un côté, le petit garçon ébouriffé turbulent de l'autre. Mais les innocentes bouclettes de Shirley Temple se retrouvent au XXI ${ }^{e}$ siècle dans le phénomène des concours de Mini Miss, qui révèlent une hyper sexualisation de la petite fille dont Temple fut une devancière involontaire.

\section{Isabelle Roussel-Gillet (Université Lille 2), « Revivance de quelques anges et super-héros "}

Isabelle Roussel-Gillet fait le lien entre la figure de l'ange et celle du super-héros dans notre culture monothéiste. En effet, plusieurs exemples montrent clairement une hybridation de l'ange et du super-héros, comme si les codes visuels et sémantiques s'entre-contaminaient, puisque les fonctions sont semblables : faire le bien, combattre le mal. Parfois l'hybridation fonctionne sur un mode ironique, parodique ou humoristique (la chute de l'ange peut être dépeinte comme un gag). Les anges, les super-héros sont-ils des messagers encore audibles? Peuvent-ils encore sauver l'humanité ? Les auteurs semblent penser que non, au vu de leur impossibilité d'empêcher le 11 septembre. L'iconicité du super-héro met le visible au premier plan : être super-héro, c'est être celui qui voit mieux, celui qui doit être vu, avec une large dose de narcissisme.

\section{Atelier 6 : « Icônes et médias »}

\section{Flavia Nascimento (Université d'État Saô Paulo, Brésil), « Icônes culturelles brésiliennes et métissage »}

Flavia Nascimento a opté pour une approche globale et panoramique pour passer en revue les icônes culturelles brésiliennes les plus marquantes. Des timbres postaux rendant hommage à la chanteuse et actrice Carmen Miranda, aux footballers, le métis Garrincha et le célèbre "roi Pelé ", iconisés par le livre de photographies Futebol-Arte, de Caio Vilela, les brésiliens se représentent eux-mêmes comme un peuple métis. Un autre exemple est le lundu, danse afro-brésilienne d'origine bantoue, créolisée, assimilée à de la sorcellerie. Malgré sa sensualité, cette danse est acceptée par la bourgeoisie blanche. Cela correspond à une certaine idée du métissage, le mythe fondateur de la société brésilienne où enfants apprennent à l'école qu'ils sont le mélange de trois races - blanche, noire et indienne. Pourtant, cette "miscégénation » s'est faite dans la douleur, comme en témoigne le racisme manifeste envers les Noirs et les Indiens.

\section{Bertrand Dicale (écrivain, journaliste), « Du bon usage de la grande dame de la chanson française "}

Bertrand Dicale s'est interrogé sur ce qui fait d'une chanteuse une grande dame de la chanson: est-ce le personnage, la voix ? Les constructions imaginaires sont riches et nombreuses autour de ce concept à la fois clair et sciemment nébuleux, subjectif, associé à une vague notion de noblesse. Juliette Gréco, par exemple, n'a pas eu un grand succès commercial, alors que son statut de grande dame de la chanson française est incontestable. L'idée sous-jacente serait que les actrices sont des femmes idéales pour 
les hommes alors que les chanteuses sont des femmes idéales pour les femmes. Quelle est la survivance de ces grandes dames de nos jours? Selon Bertand Dicale, c'est difficile à dire, car les catégories se ré-écrivent en permanence. Qui peut dire qui sera considéré comme grande dame de la chanson française parmi les chanteuses actuelles? Pour le déterminer nous avons besoin de recul sur la carrière des artistes et aussi sur notre culture populaire. Mais n'est-ce-pas une question en soi désuète ? Y-aura-t-il forcément des grandes dames actuelles, ou le phénomène est-il cantonné au passé ?

\section{Françoise Zamour (ENS Paris), « De l'acteur à l'icône, le cas Alain Delon »}

21 Selon Françoise Zamour, les images d'Alain Delon dans la publicité pour le parfum « Eau Sauvage » créent un vertige temporel depuis 2009, étant tirées du film La Piscine, de Jean-Marie Perrier, film à l'érotisme sulfureux tourné en 1968. La Piscine contribue à iconiser une conception de la masculinité. À ce titre, Delon dans ses films opère une sorte de transsubstantiation, pas seulement pour la fragilité paradoxale qu'il joue dans Rocco et ses frères, où son corps offert pour le bien de sa famille a une dimension sacrificielle, mais aussi par le passage de l'acteur à l'icône, d'une présence charnelle à une aura de saint, voire de christ.

\section{Atelier 7 : «Pratique des icônes »}

\section{Philippe Lissac (photographe, directeur de l'agence photo Godong), « Représentation du sacré dans la photographie »}

Le postulat de Philippe Lissac est que la photographie de presse est basée sur le recyclage des icônes religieuses. Par exemple, la photographie qu'on a appelée «La Madone de Bentalla" a eu l'effet miraculeux d'attirer l'attention du monde sur le conflit, tout en soulevant le problème de l'ethnocentrisme dans le fait d'appliquer des images chrétiennes hors d'Occident. La guerre représentée du côté des victimes correspond aux thèmes chrétiens de la Passion et du massacre d'innocents; les images de femmes, au thème de la Pietà. L'iconographie chrétienne impose une grille de lecture au spectateur, donne du sens à la souffrance montrée, la rend supportable. Chaque photographe doit naviguer entre usage éthique et esthétique de l'icône.

\section{Table ronde autour de L'Amérique des Images (Hazan/ Paris-Diderot, 2013)}

La culture de l'image américaine ayant dépassé les frontières de l'Amérique, il convient de se demander quelles images ont le statut d'icônes dans l'Amérique des images. Pourtant, même si le livre n'a pas été conçu avec une liste préétablie d'icônes, il a fini par comporter de nombreuses parodies d'icônes, comme Mickey ou Kennedy. Y a-t-il une cohérence des icônes ? La cohérence et l'incohérence sont souvent mêlées, comme dans la contradiction des personnes vénérées : Eliott Ness et $\mathrm{Al}$ Capone sont tous les deux des icônes. 
hème de l'icône a donc prouvé sa capacité à réunir autour d'une réflexion commune des chercheurs, étudiants et écrivains venant d'horizons infiniment variés, qui ont donné, chacun à leur façon, des outils pour analyser un monde où le visuel domine. Le visuel domine tellement qu'il peut parfois saturer notre expérience du monde, le risque étant d'accepter les messages visuels sans prendre le temps de les comprendre.

Page du colloque sur l'agenda de l'ENS :

http://www.ens.fr/actualites/agenda/article/colloque-survivances-des-icones?lang=en

Programme en PDF :

http://www.ens.fr/IMG/image/actus/2014/agenda/Avril/Programme_survivances.pdf

\section{AUTHOR}

JULIETTE MÉLIA

Université Paris 7 